\title{
Interactive effects of rice bran compost and chemical fertilizers on macronutrients, oil and protein content in sunflower (Helianthus annuus L.)
}

\author{
M. Alauddin'1, G.M. Mohsin²* A.H.M.Z. Ali3 ${ }^{2}$ and M.K. Rahman³
}

Received 19 August 2020, Revised 14 December 2020, Accepted 24 December 2020, Published online 31 December 2020

\section{A B S T R A C T}

A field experiment was conducted at the research farm of Charfasson Govt. College, Bhola, Bangladesh in rabi season in 2015-2016 to evaluate the impact of conjunctive use of chemical fertilizers with rice bran on concentration, uptake and seed quality of sunflower $c v$. BARI-2 (Keroni-2). The experiment was laid out in the randomized complete block design (RCBD) having sixteen treatments with three replications. The size of the plots were $3 \mathrm{~m} \mathrm{x} 2$ m. Treatments were $\mathrm{T}_{1}$ Control (- RB and -NPK), $\mathrm{T}_{2}: 2.5 \mathrm{t} \mathrm{RB} \mathrm{ha}^{-1}, \mathrm{~T}_{3}: 5.0$ t RB ha-1, $\mathrm{T}_{4}: 7.5 \mathrm{t}$ RB ha-1, $\mathrm{T}_{5}: \mathrm{N}_{40} \mathrm{P}_{30} \mathrm{~K}_{50} \mathrm{~kg} \mathrm{ha}^{-1}, \mathrm{~T}_{6}: \mathrm{N}_{80} \mathrm{P}_{60} \mathrm{~K}_{100} \mathrm{~kg} \mathrm{ha}^{-1}, \mathrm{~T}_{7}: \mathrm{N}_{120} \mathrm{P}_{90} \mathrm{~K}_{150} \mathrm{~kg} \mathrm{ha}^{-1}, \mathrm{~T}_{8}: 2.5 \mathrm{t} \mathrm{RB} \mathrm{ha}^{-1}+$ $\mathrm{N}_{40} \mathrm{P}_{30} \mathrm{~K}_{50} \mathrm{~kg} \mathrm{ha}^{-1}, \mathrm{~T}_{9}: 2.5$ t RB ha-1 $+\mathrm{N}_{80} \mathrm{P}_{60} \mathrm{~K}_{100} \mathrm{~kg} \mathrm{ha}^{-1}, \mathrm{~T}_{10}: 2.5$ t RB ha-1 $+\mathrm{N}_{120} \mathrm{P}_{90} \mathrm{~K}_{150} \mathrm{~kg}$ ha 1, $\mathrm{T}_{11}: 5.0$ t RB ha-1 $+\mathrm{N}_{40} \mathrm{P}_{30} \mathrm{~K}_{50} \mathrm{~kg} \mathrm{ha}^{-1}, \mathrm{~T}_{12}$ : 5.0 t RB ha-1 $+\mathrm{N}_{80} \mathrm{P}_{60} \mathrm{~K}_{100} \mathrm{~kg} \mathrm{ha}^{-1}, \mathrm{~T}_{13}: 5.0$ t RB ha${ }^{1}+\mathrm{N}_{120} \mathrm{P}_{90} \mathrm{~K}_{150} \mathrm{~kg} \mathrm{ha}^{-1}, \mathrm{~T}_{14}: 7.5$ t RB ha-1 $+\mathrm{N}_{40} \mathrm{P}_{30} \mathrm{~K}_{50} \mathrm{~kg} \mathrm{ha}^{-1}, \mathrm{~T}_{15}: 7.5 \mathrm{t} \mathrm{RB} \mathrm{ha}^{-1}+\mathrm{N}_{80} \mathrm{P}_{60} \mathrm{~K}_{100} \mathrm{~kg}$ $\mathrm{ha}^{-1}, \mathrm{~T}_{16}: 7.5 \mathrm{t} \mathrm{RB} \mathrm{ha}{ }^{-1}+\mathrm{N}_{120} \mathrm{P}_{90} \mathrm{~K}_{150} \mathrm{~kg} \mathrm{ha}^{-1}$. Results showed that the concentration, uptake and quality of seeds (oil and protein) of the crop increased with increasing rate of the amendments significantly $(\mathrm{P}<0.05)$ over the control and the variation between the treatments were also significant irrespective of the sources of amendments in most of the cases. Generally, combination of the treatments showed better performance than their individual application. Maximum values of NPKS concentration (\%) in different organs of sunflower were 1.22, $0.35,1.90,0.18$ for stem; $1.17,0.35,2.41,0.16$ for root; $3.98,0.43,4.28$, 0.24 for leaf, 1.04, $0.65,3.00,0.22$ for petiole; 2.16, $0.58,2.21,0.26$ for inflorescence and $5.24,0.83,1.60,0.47$ for seed measured in treatments 5.0 t RB ha-1 $+\mathrm{N}_{120} \mathrm{P}_{90} \mathrm{~K}_{150} \mathrm{~kg} \mathrm{ha}^{-1}$ and $7.5 \mathrm{t} \mathrm{RB} \mathrm{ha} \mathrm{h}^{-1}+\mathrm{N}_{120} \mathrm{P}_{90} \mathrm{~K}_{150} \mathrm{~kg} \mathrm{ha}^{-1}$ in most of the cases. However, their uptake pattern also followed the same trend as in concentration and the highest values were found in those treatments in most of the cases. Significantly $(\mathrm{P}<0.05 \%)$ the highest content of oil $(51.1 \%)$ in seed was measured in the treatment 5.0 t RB ha-1 $+\mathrm{N}_{120} \mathrm{P}_{90} \mathrm{~K}_{150} \mathrm{~kg} \mathrm{ha}^{-1}$ and protein (33.9\%) was found in the treatment 5.0 t RB ha-1 $+\mathrm{N}_{80} \mathrm{P}_{60} \mathrm{~K}_{100} \mathrm{~kg} \mathrm{ha}^{-1}$. Their lowest values were found in control for oil and in $2.5 \mathrm{t} \mathrm{RB} \mathrm{ha-1}$ for protein, which was lower than control treatment. The overall findings of this study indicated that rice bran in combination with chemical fertilizers could be applied to achieve better concentration and uptake in different organs, oil and protein content in seeds of sunflower.

Keywords: Oil and protein, Rice bran and chemical fertilizers, Sunflower, Uptake.

${ }^{1}$ Department of Soil Science, Charfasson Govt. College, Bhola, Bangladesh.

${ }^{2}$ Department of Agriculture, Noakhali Science and Technology University, Sonapur, Noakhali-3814, Bangladesh.

${ }^{3}$ Department of Soil, Water and Environment, University of Dhaka, Dhaka-1000, Bangladesh.

*Corresponding author's email: mohsinbreeder97@gmail.com (G.M. Mohsin)

Cite this article as: Alauddin, M., Mohsin, G.M., Ali, A.H.M.Z. and Rahman, M.K. 2020. Interactive effects of rice bran compost and chemical fertilizers on macronutrients, oil and protein content in sunflower (Helianthus annuus L.). Int. J. Agril. Res. Innov. Tech. 10(2): 91-99. https://doi.org/10.3329/ijarit.v10i2.51582

\section{Introduction}

Sunflower (Helianthus annuus L.) is one of the most important oil seed crops in the world's oil seed production, because it offers advantages in crop rotation systems, such as high adaptation capability, suitability to mechanization, low labor needs and easy cultivated and grown in different conditions and soils. In addition, it is one of the crops, which have high availability to planting and produce high yield under stress such as (drought, salinity or temperature). The oil 
extracted (48-53\%) is edible from this crop; about $80 \%$ of the oil is used for edible purpose and rest being non-edible, used for industrial purposes.

Agriculture plays an important role in economy of developing countries like Pakistan (Badar and Qureshi, 2014), Bangladesh and so on. However, rapid crop production with inappropriate farming practices deteriorate organic matter in soil, which results in decreased microbial activity that eventually affect its physical, chemical and biological conditions which lead to decline in land productivity and crops yields. To solve this problem, synthetic fertilizers were always thought to be a better way to improve the soil fertility and crop productivity but unfortunately, the excessive use of these creates a number of serious environmental and health risk (Badar and Qureshi, 2014). Agro-chemicals deteriorate soil health and environment got pollute. Problems associated with continuous use of chemical fertilizers included nutrient imbalance, increased soil acidity, degradation in soil physical properties and loss of organic matter. Hence, the tendency to supply all plant nutrients through chemical fertilizer should be reconsidered in the future because of the deleterious effect on soil productivity on a long-term basis (Moyin-Jesu, 2015). To minimize these hazards, naturally occurring organic fertilizers, namely animal and plant manures, fall residues, and food and urban wastes are better alternate of commercially available fertilizers. Reports proved that organic farming improves soil composition, fertility, and soil fauna, which in the long run have a beneficial effect on crop production (Badar and Qureshi, 2014). Leguminous materials and rice bran (RB) supplied mainly N, P, K, Zn, Fe, Cu, Mn and B to the soil those NPK 15-15-15 fertilizer did not possess. The organic materials applied (wood ash, rice bran and so firth) have beneficial residual effects on soil properties which are in line with growing concern of using environment friendly fertilizer (Moyin-Jesu, 2015). Mahrous et al. (2014) reported that different organic nutrient management practices have been found to be technically and financially beneficial. Adding nutrient in the form of organic fertilizers has many advantages, e.g. they enhance soil biological activity, which improves nutrient mobilization, enhance root growth due to better soil structure, release nutrients slowly and contribute to the industrial pool of organic $\mathrm{N}$ and $\mathrm{P}$ in the soil. Also using organic fertilizers reducing $\mathrm{N}$ leaching loss and $\mathrm{P}$ fixation; they can also supply micronutrients, increase the organic matter content of the soil, therefore improving the exchange capacity of nutrients, increasing soil water retention, promoting soil aggregates.
Sunflower is highly productive in sandy loam as well as a clay loam soil. Therefore, farmers could cultivate this crop widely both in rabi and kharif seasons in the coastal areas of Bangladesh. Moreover, it reduces climate change vulnerability by emission of large amount of $\mathrm{CO}_{2}$ (Mahapatra and Sharma, 1989). For higher productivity and sustainability, integrated use of organic and inorganic sources of nutrient is very important (Rasool et al., 2013). Keeping these aspects in view, the present investigation was carried out to examine the impact of conjunctive use of rice bran and chemical fertilizer on concentration, uptake and seed quality of sunflower (Helianthus annuus L.).

\section{Materials and Methods}

A field study was carried out at the research farm of Charfasson Govt. College, Bhola, Bangladesh during rabi season in 2015-2016. Soil samples (o$15 \mathrm{~cm}$ depth) were collected from the research farm. The sample was air dried, ground and sieved through $3 \mathrm{~mm}$ sieve for chemical analysis and $2 \mathrm{~mm}$ sieve for physical analysis and kept in polyethylene bags. The soil had found $\mathrm{pH}$ of 8.36 (1: $2.5 \mathrm{w} / \mathrm{v} \mathrm{H}_{2} \mathrm{O}$ ), organic carbon $0.63 \%$ (Wet oxidation method, Walkley and Black, 1934), available nitrogen $0.24 \%$ (Kjeldahl extraction, Marr and Cresser, 1983), available phosphorus 0.06\% (Jackson, 1958), available potassium 1.23\% (Pratt, 1965), available sulfur $0.15 \%$ (Bardsley and Lancaster, 1965), sand 12.3\%, silt $51.34 \%$ and clay $36.36 \%$, textural class- silty clay loam (Bouyoucos, 1962), the maximum water retentive capacity was $37 \%$, respectively. Seeds were collected from BADC, Barishal. The variety of seed was BARI-2 (Keroni-2). The experiment was laid out in a randomized complete block design (RCBD) having sixteen treatments with three replications. The unit plot size was $3.0 \mathrm{~m} \mathrm{x}$ $2.0 \mathrm{~m}$ and total plots were 48. Treatments were $\mathrm{T}_{1}$ Control (-RB and -NPK), $\mathrm{T}_{2}: 2.5$ t RB ha-1, $\mathrm{T}_{3}$ :

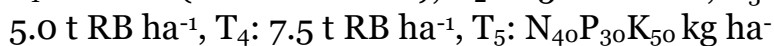
${ }^{1}, \mathrm{~T}_{6}: \mathrm{N}_{80} \mathrm{P}_{60} \mathrm{~K}_{100} \mathrm{~kg} \mathrm{ha}^{-1}, \mathrm{~T}_{7}: \mathrm{N}_{120} \mathrm{P}_{90} \mathrm{~K}_{150} \mathrm{~kg} \mathrm{ha}^{-1}, \mathrm{~T}_{8}$ : 2.5 t RB ha-1 $+\mathrm{N}_{40} \mathrm{P}_{30} \mathrm{~K}_{50} \mathrm{~kg} \mathrm{ha}^{-1}, \mathrm{~T}_{9}: 2.5$ t RB ha-1 $+\mathrm{N}_{80} \mathrm{P}_{60} \mathrm{~K}_{100} \mathrm{~kg} \mathrm{ha} \mathrm{ha}^{-1}, \mathrm{~T}_{10}: 2.5 \mathrm{t} \mathrm{RB} \mathrm{ha-1}+$ $\mathrm{N}_{120} \mathrm{P}_{90} \mathrm{~K}_{150} \mathrm{~kg} \mathrm{ha}^{-1}, \mathrm{~T}_{11}: 5.0$ t RB ha ${ }^{-1}+\mathrm{N}_{40} \mathrm{P}_{30} \mathrm{~K}_{50}$ $\mathrm{kg} \mathrm{ha}^{-1}, \mathrm{~T}_{12}$ : 5.0 t RB ha-1 $+\mathrm{N}_{80} \mathrm{P}_{60} \mathrm{~K}_{100} \mathrm{~kg} \mathrm{ha}^{-1}, \mathrm{~T}_{13}$ : 5.0 t RB ha-1 $+\mathrm{N}_{120} \mathrm{P}_{90} \mathrm{~K}_{150} \mathrm{~kg} \mathrm{ha}^{-1}, \mathrm{~T}_{14}: 7.5$ t RB $\mathrm{ha}^{-1}+\mathrm{N}_{40} \mathrm{P}_{30} \mathrm{~K}_{50} \mathrm{~kg} \mathrm{ha}^{-1}, \mathrm{~T}_{15}: 7.5 \mathrm{t} \mathrm{RB} \mathrm{ha}{ }^{-1}+$ $\mathrm{N}_{80} \mathrm{P}_{60} \mathrm{~K}_{100} \mathrm{~kg} \mathrm{ha}^{-1}$ and $\mathrm{T}_{16}: 7.5 \mathrm{t}$ RB ha-1 + $\mathrm{N}_{120} \mathrm{P}_{90} \mathrm{~K}_{150} \mathrm{~kg} \mathrm{ha}^{-1}$.

The doses were selected according to the Fertilizer Recommendation Guide of Bangladesh Agricultural Research Council (BARC, 2012). At the time of initial land preparation, rice bran was applied and at final land preparation, $\mathrm{N}, \mathrm{P}$ and $\mathrm{K}$ were applied as urea, triple super phosphate and 
muriate of potash, respectively. Seeds were sown on 29 December, 2015. Sixty seeds were sown in each plot. Length between row to row was $40 \mathrm{~cm}$ and width between seed to seed was $25 \mathrm{~cm}$. Intercultural practices i.e. weeding, spading, fencing, pesticide etc. were applied as per when needed. Finally, plants were harvested after 90 days of sowing of seeds at the period of maturity. Different organs of sunflower plants viz., stem, root, leaf, petiole, inflorescence and seed were collected and dried in an oven at temperature of $65^{\circ} \mathrm{C}$. The dry weight of different parameters and seed weights were measured and those were kept in paper bags separately. The uptake of nutrients by different parts of sunflower plant was worked out by multiplying the nutrient concentration and dry matter yield of the plant parts. Estimation of oil content (\%) in the seed sample was done by Soxhlet Fat Extraction method evolved by (AOAC, 1990). Seed protein content was calculated by multiplying the $\mathrm{N}$ content of seed with a factor of 6.25. Analysis of variance was done with the help of SPSS program and the mean differences among different treatments were evaluated by LSD test at 5\% level.

\section{Results and Discussion}

\section{(a) Concentration and uptake of NPKS in root}

Effects of rice bran and NPK fertilizers on NPKS concentration and uptake of root of sunflower were determined. The results showed that both concentration and uptake of NPKS of root increased significantly $(\mathrm{P}<0.05)$ due to application of various combinations of rice bran and NPK fertilizers over the control (Table 1). The treatments generally showed an increase in both the concentrations and uptakes of NPKS in sunflower root with increasing rates of both rice bran and NPK fertilizers. The values of concentration and uptake of the nutrients in root of sunflower when compared between the treatments, in most of the cases, identical results were obtained. Rice bran showed better results in nitrogen and sulfur concentration of root than fertilizer when applied alone. But, phosphorus and potassium concentrations in root of sunflower were found to be better in fertilizer treated plants. The ranges of nitrogen concentration and uptake were found to be 0.52 to $1.17 \%$ and 5.2 to $187.2 \mathrm{mg}$ plant $^{-1}$ root, respectively.

Table 1. Effects of rice bran compost and NPK fertilizers on the concentration and uptake of NPKS in root of sunflower.

\begin{tabular}{|c|c|c|c|c|c|c|c|c|}
\hline \multirow[t]{2}{*}{ Treatments } & \multicolumn{2}{|c|}{ Nitrogen } & \multicolumn{2}{|c|}{ Phosphorus } & \multicolumn{2}{|c|}{ Potassium } & \multicolumn{2}{|c|}{ Sulfur } \\
\hline & $\begin{array}{l}\text { Conc. } \\
(\%)\end{array}$ & $\begin{array}{l}\text { Uptake } \\
(\mathrm{mg} \\
\left.\text { plant }^{-1}\right)\end{array}$ & $\begin{array}{l}\text { Conc. } \\
\text { (\%) }\end{array}$ & $\begin{array}{l}\text { Uptake } \\
\text { (mg } \\
\left.\text { plant }^{-1}\right)\end{array}$ & $\begin{array}{c}\text { Conc. } \\
(\%)\end{array}$ & $\begin{array}{l}\text { Uptake } \\
(\mathrm{mg} \\
\left.\text { plant }^{-1}\right)\end{array}$ & $\begin{array}{c}\text { Conc. } \\
(\%)\end{array}$ & $\begin{array}{l}\text { Uptake } \\
\text { (mg } \\
\left.\text { plant }^{-1}\right)\end{array}$ \\
\hline $\mathrm{T}_{1}$ : Control (-RB and -NPK) & 0.52 & 5.2 & 0.09 & 0.9 & 0.89 & 8.9 & 0.07 & 0.7 \\
\hline $\mathrm{T}_{2}: 2.5 \mathrm{t} \mathrm{RB} \mathrm{ha}^{-1}$ & 0.67 & 13.4 & 0.13 & 2.6 & 1.18 & 23.6 & 0.07 & 1.4 \\
\hline $\mathrm{T}_{3}: 5.0$ t RB ha-1 & 0.68 & 22.4 & 0.15 & 5.0 & 1.27 & 41.9 & 0.14 & 4.6 \\
\hline $\mathrm{T}_{4}: 7.5 \mathrm{t} \mathrm{RB} \mathrm{ha}^{-1}$ & 0.83 & 41.5 & 0.15 & 7.5 & 1.46 & 73.0 & 0.16 & 8.0 \\
\hline $\mathrm{T}_{5}: \mathrm{N}_{40} \mathrm{P}_{30} \mathrm{~K}_{50} \mathrm{~kg} \mathrm{ha}^{-1}$ & 0.65 & $45 \cdot 5$ & 0.12 & 8.4 & 1.54 & 107.8 & 0.09 & 6.3 \\
\hline $\mathrm{T}_{6}: \mathrm{N}_{80} \mathrm{P}_{60} \mathrm{~K}_{100} \mathrm{~kg} \mathrm{ha}^{-1}$ & 0.67 & 48.2 & 0.19 & 13.7 & 1.58 & 113.8 & 0.09 & 6.5 \\
\hline $\mathrm{T}_{7}: \mathrm{N}_{120} \mathrm{P}_{90} \mathrm{~K}_{15 \mathrm{o}} \mathrm{kg} \mathrm{ha}^{-1}$ & 0.68 & 54.4 & 0.25 & 20.0 & 1.94 & 155.2 & 0.08 & 6.4 \\
\hline $\begin{array}{l}\text { T8: } 2.5 \text { t } \mathrm{RB} \mathrm{ha}^{-1} \\
+\mathrm{N}_{40} \mathrm{P}_{30} \mathrm{~K}_{50} \mathrm{~kg} \mathrm{ha}^{-1}\end{array}$ & $0.5^{2}$ & 42.5 & 0.19 & 19.2 & 2.03 & 205.0 & 0.09 & 9.1 \\
\hline $\begin{array}{l}\mathrm{T}_{9}: 2.5 \text { t RB ha-1 } \\
+\mathrm{N}_{80} \mathrm{P}_{60} \mathrm{~K}_{100} \mathrm{~kg} \mathrm{ha}^{-1}\end{array}$ & 0.71 & $97 \cdot 3$ & 0.23 & 31.5 & 2.07 & 283.6 & 0.08 & 11.0 \\
\hline $\begin{array}{l}\mathrm{T}_{10}: 2.5 \mathrm{t} \mathrm{RB} \mathrm{ha}{ }^{-1} \\
+\mathrm{N}_{120} \mathrm{P}_{90} \mathrm{~K}_{150} \mathrm{~kg} \mathrm{ha}^{-1}\end{array}$ & 1.17 & 161.5 & 0.25 & $34 \cdot 5$ & 2.11 & 291.2 & 0.13 & 17.9 \\
\hline $\begin{array}{l}\mathrm{T}_{11}: 5.0 \text { t } \mathrm{RB} \mathrm{ha}^{-1} \\
+\mathrm{N}_{40} \mathrm{P}_{30} \mathrm{~K}_{50} \mathrm{~kg} \mathrm{ha}^{-1}\end{array}$ & 0.93 & 130.2 & 0.21 & 29.4 & 2.41 & 237.4 & 0.10 & 14.0 \\
\hline $\begin{array}{l}\mathrm{T}_{12}: 5.0 \text { t RB ha } \\
+\mathrm{N}_{80} \mathrm{P}_{60} \mathrm{~K}_{100} \mathrm{~kg} \mathrm{ha}^{-1}\end{array}$ & 0.69 & 73.8 & 0.20 & 21.4 & 1.88 & 201.2 & 0.09 & 9.6 \\
\hline $\begin{array}{l}\mathrm{T}_{13}: 5.0 \text { t } \mathrm{RB} \mathrm{ha}^{-1} \\
+\mathrm{N}_{120} \mathrm{P}_{90} \mathrm{~K}_{150} \mathrm{~kg} \mathrm{ha}^{-1}\end{array}$ & 1.17 & 187.2 & 0.35 & 56.0 & 2.15 & 344.0 & 0.12 & 19.2 \\
\hline $\begin{array}{l}\mathrm{T}_{14}: 7.5 \text { t RB ha-1 } \\
+\mathrm{N}_{40} \mathrm{P}_{30} \mathrm{~K}_{50} \mathrm{~kg} \mathrm{ha}^{-1}\end{array}$ & 0.92 & 135.2 & 0.21 & 30.9 & 2.05 & 301.4 & 0.10 & 14.7 \\
\hline $\begin{array}{l}\mathrm{T}_{15}: 7.5 \text { t RB ha-1 } \\
+\mathrm{N}_{80} \mathrm{P}_{60} K_{100} \mathrm{~kg} \mathrm{ha}^{-1}\end{array}$ & 0.98 & 153.9 & 0.17 & 26.7 & 1.71 & 268.5 & 0.10 & $15 \cdot 7$ \\
\hline $\begin{array}{l}\mathrm{T}_{16}: 7.5 \text { t RB ha-1 } \\
+\mathrm{N}_{120} \mathrm{P}_{90} \mathrm{~K}_{150} \mathrm{~kg} \mathrm{ha}^{-1}\end{array}$ & 0.83 & 132.8 & 0.23 & 36.8 & 1.96 & 313.6 & 0.11 & 17.6 \\
\hline LSD at 5\% & 0.16 & 7.49 & 0.04 & 5.02 & 0.44 & $15 \cdot 37$ & 0.05 & 1.75 \\
\hline
\end{tabular}


The highest value of nitrogen concentration was recorded in treatments, $2.5 \mathrm{t} \mathrm{RB} \mathrm{ha-1}+\mathrm{N}_{120} \mathrm{P}_{90} \mathrm{~K}_{150}$ $\mathrm{kg} \mathrm{ha}^{-1}$ and 5.0 t RB ha-1 $+\mathrm{N}_{120} \mathrm{P}_{90} \mathrm{~K}_{150} \mathrm{~kg} \mathrm{ha}^{-1}$ but that of uptake was observed in 5.0 t $\mathrm{RB} \mathrm{ha}^{-1}+$ $\mathrm{N}_{120} \mathrm{P}_{90} \mathrm{~K}_{150} \mathrm{~kg} \mathrm{ha}^{-1}$ treatment. Like nitrogen, concentration and uptake of phosphorus in root of sunflower ranged from 0.09 to $0.35 \%$ and 0.9 to $56.0 \mathrm{mg}$ plant $^{-1}$ root, respectively (Table 1). Both the highest values were observed in the same treatment, 5.0 t RB ha-1 $+\mathrm{N}_{120} \mathrm{P}_{90} \mathrm{~K}_{150} \mathrm{~kg} \mathrm{ha}^{-}$ 1. Potassium concentration and uptake of sunflower root were found to be 0.89 to 2.41 and 8.9 to $344.0 \mathrm{mg}$ plant $^{-1}$ root, respectively (Table 3). The highest values were recorded in treatments, 5.0 t RB ha-1 $+\mathrm{N}_{40} \mathrm{P}_{30} \mathrm{~K}_{50} \mathrm{~kg} \mathrm{ha}^{-1}$ and 5.0 t CD ha-1 $+\mathrm{N}_{120} \mathrm{P}_{90} \mathrm{~K}_{150} \mathrm{~kg} \mathrm{ha}^{-1}$, respectively. Sulfur concentration and uptake of root ranged from 0.07 to $0.16 \%$ and 0.7 to $19.2 \mathrm{mg}$ plant $^{-1}$ root, respectively (Table 1 ). The highest recorded values were observed in treatments, $7.5 \mathrm{t} \mathrm{RB} \mathrm{ha-1}$ and 5.0 $\mathrm{t}$ RB ha ${ }^{-1}+\mathrm{N}_{120} \mathrm{P}_{90} \mathrm{~K}_{150} \mathrm{~kg} \mathrm{ha}{ }^{-1}$, respectively.

\section{(b) Concentration and uptake of NPKS in stem}

Effects of rice bran and NPK fertilizers on concentration and uptake of NPKS of stem of sunflower were determined. The results showed that both concentration and uptake of NPKS of stem increased significantly $(\mathrm{P}<0.05)$ due to application of various combinations of rice bran and NPK fertilizers over the control (Table 2). The values of concentration and uptake of the nutrients in stem of sunflower when compared between the treatments, in most of the cases, identical results were obtained. Nitrogen, phosphorus and potassium concentrations in stem were comparatively more effective in inorganic fertilizers treated plants than rice bran treated ones. An opposite effect was observed in case of sulfur concentration in stem. Nitrogen concentration and uptake varied from 1.07 to $1.22 \%$ and 85.6 to $787.4 \mathrm{mg}_{\text {plant }}^{-1}$ stem, respectively.

Table 2. Effects of rice bran compost and NPK fertilizers on the concentration and uptake of NPKS in stem of sunflower.

\begin{tabular}{|c|c|c|c|c|c|c|c|c|}
\hline \multirow[t]{2}{*}{ Treatments } & \multicolumn{2}{|c|}{ Nitrogen } & \multicolumn{2}{|c|}{ Phosphorus } & \multicolumn{2}{|c|}{ Potassium } & \multicolumn{2}{|c|}{ Sulfur } \\
\hline & $\begin{array}{l}\text { Conc. } \\
(\%)\end{array}$ & $\begin{array}{l}\text { Uptake } \\
\left(\mathrm{mg}^{-1}\right. \\
\left.\text { plant }^{-1}\right)\end{array}$ & $\begin{array}{c}\text { Conc. } \\
\text { (\%) }\end{array}$ & $\begin{array}{l}\text { Uptake } \\
\left(\mathrm{mg}^{-1}\right. \\
\left.\text { plant }^{-1}\right)\end{array}$ & $\begin{array}{c}\text { Conc. } \\
(\%)\end{array}$ & $\begin{array}{l}\text { Uptake } \\
\text { (mg } \\
\left.\text { plant }^{-1}\right)\end{array}$ & $\begin{array}{c}\text { Conc. } \\
(\%)\end{array}$ & $\begin{array}{l}\text { Uptake } \\
\left(\mathrm{mg}^{-1}\right. \\
\left.\text { plant }^{-1}\right)\end{array}$ \\
\hline $\mathrm{T}_{1}$ : Control (-RB and -NPK) & 1.07 & 85.6 & 0.11 & 8.80 & 0.62 & 49.6 & 0.07 & 5.6 \\
\hline $\mathrm{T}_{2}: 2.5$ t RB ha-1 & 1.10 & 117.7 & 0.20 & 21.40 & 1.18 & 126.3 & 0.10 & 10.7 \\
\hline $\mathrm{T}_{3}: 5.0$ t RB ha-1 & 1.18 & 156.9 & 0.22 & 29.26 & 1.40 & 186.2 & 0.12 & 16.0 \\
\hline $\mathrm{T}_{4}: 7 \cdot 5 \mathrm{t} \mathrm{RB} \mathrm{ha}^{-1}$ & 1.20 & 192.0 & 0.24 & 38.40 & 1.50 & 240.0 & 0.15 & 24.0 \\
\hline $\mathrm{T}_{5}: \mathrm{N}_{40} \mathrm{P}_{30} \mathrm{~K}_{50} \mathrm{~kg} \mathrm{ha}^{-1}$ & 1.21 & 378.7 & 0.28 & 87.60 & 1.56 & 488.3 & 0.09 & 28.2 \\
\hline $\mathrm{T}_{6}: \mathrm{N}_{80} \mathrm{P}_{60} \mathrm{~K}_{100} \mathrm{~kg} \mathrm{ha}^{-1}$ & 1.13 & 392.1 & 0.27 & 93.70 & 1.59 & 551.7 & 0.10 & 34.7 \\
\hline $\mathrm{T}_{7}: \mathrm{N}_{120} \mathrm{P}_{90} \mathrm{~K}_{15 \mathrm{o}} \mathrm{kg} \mathrm{ha}^{-1}$ & 1.22 & 610.0 & 0.23 & 115.00 & 1.66 & 830.0 & 0.13 & 65.0 \\
\hline $\begin{array}{l}\text { T8: } 2.5 \mathrm{t} \mathrm{RB} \mathrm{ha}^{-1} \\
+\mathrm{N}_{40} \mathrm{P}_{30} \mathrm{~K}_{50} \mathrm{~kg} \mathrm{ha}^{-1}\end{array}$ & 1.14 & 501.6 & 0.24 & 105.60 & 152 & 668.8 & 0.17 & 74.8 \\
\hline $\begin{array}{l}\mathrm{T}_{9}: 2.5 \mathrm{t} \mathrm{RB} \mathrm{ha}^{-1} \\
+\mathrm{N}_{80} \mathrm{P}_{60} \mathrm{~K}_{100} \mathrm{~kg} \mathrm{ha}^{-1}\end{array}$ & 1.16 & 541.7 & 0.20 & 93.40 & 1.49 & 695.8 & 0.15 & 70.1 \\
\hline $\begin{array}{l}\mathrm{T}_{10}: 2.5 \mathrm{t} \mathrm{RB} \mathrm{ha}^{-1} \\
+\mathrm{N}_{120} \mathrm{P}_{90} \mathrm{~K}_{150} \mathrm{~kg} \mathrm{ha}^{-1}\end{array}$ & 1.11 & 502.8 & 0.24 & 108.70 & 1.68 & 761.0 & 0.18 & 81.5 \\
\hline $\begin{array}{l}\mathrm{T}_{11}: 5.0 \mathrm{t} \mathrm{RB} \mathrm{ha} \\
+\mathrm{N}_{40} \mathrm{P}_{30} \mathrm{~K}_{50} \mathrm{~kg} \mathrm{ha}^{-1}\end{array}$ & 1.22 & 497.5 & 0.22 & 86.50 & 1.48 & 581.6 & 0.14 & 55.0 \\
\hline $\begin{array}{l}\mathrm{T}_{12}: 5.0 \text { t RB ha } \\
+\mathrm{N}_{80} \mathrm{P}_{60} \mathrm{~K}_{100} \mathrm{~kg} \mathrm{ha}^{-1}\end{array}$ & 1.21 & 629.2 & 0.28 & 145.60 & 1.72 & 894.4 & 0.16 & 83.2 \\
\hline $\begin{array}{l}\mathrm{T}_{13}: 5.0 \text { t RB ha-1 } \\
+\mathrm{N}_{120} \mathrm{P}_{90} \mathrm{~K}_{150} \mathrm{~kg} \mathrm{ha}^{-1}\end{array}$ & 1.16 & 664.7 & 0.35 & 200.60 & 1.74 & 997.0 & 0.17 & 97.4 \\
\hline $\begin{array}{l}\mathrm{T}_{14}: 7.5 \mathrm{t} \mathrm{RB} \mathrm{ha} \mathrm{R}^{-1} \\
+\mathrm{N}_{40} \mathrm{P}_{30} \mathrm{~K}_{50} \mathrm{~kg} \mathrm{ha}^{-1}\end{array}$ & 1.10 & 528.0 & 0.29 & 139.20 & 1.90 & 912.0 & 0.14 & 67.2 \\
\hline $\begin{array}{l}\mathrm{T}_{15}: 7.5 \mathrm{t} \mathrm{RB} \mathrm{ha}^{-1} \\
+\mathrm{N}_{80} \mathrm{P}_{60} \mathrm{~K}_{100} \mathrm{~kg} \mathrm{ha}^{-1}\end{array}$ & 1.14 & 749.0 & 0.30 & 197.10 & 1.52 & 998.6 & 0.17 & 111.7 \\
\hline $\begin{array}{l}\mathrm{T}_{16}: 7.5 \mathrm{t} \mathrm{RB} \mathrm{ha}^{-1} \\
+\mathrm{N}_{120} \mathrm{P}_{90} \mathrm{~K}_{150} \mathrm{~kg} \mathrm{ha}^{-1}\end{array}$ & 1.17 & 787.4 & 0.25 & 168.30 & 1.48 & 996.0 & 0.16 & 107.7 \\
\hline LSD at $5 \%$ & 0.10 & 21.03 & 0.08 & 12.09 & 0.21 & 48.67 & 0.68 & 9.13 \\
\hline
\end{tabular}

The highest values were recorded in treatments, $\mathrm{N}_{120} \mathrm{P}_{90} \mathrm{~K}_{150} \mathrm{~kg} \mathrm{ha}^{-1}$ and 5.0 t RB ha-1 $+\mathrm{N}_{40} \mathrm{P}_{30} \mathrm{~K}_{50}$ $\mathrm{kg} \mathrm{ha}{ }^{-1}$, and $7.5 \mathrm{t} \mathrm{RB} \mathrm{ha-1}+\mathrm{N}_{120} \mathrm{P}_{90} \mathrm{~K}_{150} \mathrm{~kg} \mathrm{ha}^{-1}$, respectively. Phosphorus concentration and uptake ranged from 0.11 to $0.35 \%$ and 8.8 to $200.6 \mathrm{mg} \mathrm{plant}^{-1}$ stem, respectively (Table 2). The highest values of concentration and uptake of phosphorus were recorded in the same treatment, 5.0 t RB ha-1 $+\mathrm{N}_{120} \mathrm{P}_{90} \mathrm{~K}_{150} \mathrm{~kg} \mathrm{ha}^{-1}$. Potassium concentration and uptake varied from 0.62 to $1.90 \%$ and 49.6 to $998.6 \mathrm{mg} \mathrm{plant}^{-1}$ stem, respectively (Table 2). The maximum values of concentration and uptake were observed in treatments, $7.5 \mathrm{t} \mathrm{RB} \mathrm{ha}{ }^{-1}+\mathrm{N}_{40} \mathrm{P}_{30} \mathrm{~K}_{50} \mathrm{~kg} \mathrm{ha}^{-1}$ and 
7.5 t RB ha-1 $+\mathrm{N}_{80} \mathrm{P}_{60} \mathrm{~K}_{100} \mathrm{~kg} \mathrm{ha}^{-1}$, respectively. Similarly, sulfur concentration and uptake also varied from 0.07 to $0.18 \%$ and 5.6 to $111.7 \mathrm{mg}$ plant $^{-1}$ stem, respectively (Table 2). The highest values were recorded in treatments, $2.5 \mathrm{t} \mathrm{RB} \mathrm{ha}^{-1}$ $+\mathrm{N}_{120} \mathrm{P}_{90} \mathrm{~K}_{150} \mathrm{~kg} \mathrm{ha}{ }^{-1}$ and $7.5 \mathrm{t} \mathrm{RB} \mathrm{ha}{ }^{-1}+$ $\mathrm{N}_{80} \mathrm{P}_{60} \mathrm{~K}_{100} \mathrm{~kg} \mathrm{ha}^{-1}$, respectively.

\section{(c) Concentration and uptake of NPKS in leaf}

Treatments of rice bran and NPK fertilizers, on concentration and uptake of NPKS in leaf were measured. Treatments showed significantly $(\mathrm{P}<0.05)$ positive effects with doses of rice bran and NPK fertilizers on nutrients concentrations of NPKS in sunflower leaf over the control (Table 3). But comparison between the treatments showed identical results. Between rice bran and fertilizers, rice bran played better role than inorganic fertilizers in case of only sulfur concentration. Concentration and uptake of nitrogen and phosphorus in leaf of sunflower varied from 2.18 to 3.98 and 0.28 to $0.43 \%$; and 152.6 to 903.5 and 19.6 to $97.6 \mathrm{mg}$ plant $^{-1}$ leaf of both nitrogen and phosphorus, respectively.

Table 3. Effects of rice bran compost and NPK fertilizers on the concentration and uptake of NPKS in leaf of sunflower.

\begin{tabular}{|c|c|c|c|c|c|c|c|c|}
\hline \multirow[t]{2}{*}{ Treatments } & \multicolumn{2}{|c|}{ Nitrogen } & \multicolumn{2}{|c|}{ Phosphorus } & \multicolumn{2}{|c|}{ Potassium } & \multicolumn{2}{|l|}{ Sulfur } \\
\hline & $\begin{array}{l}\text { Conc. } \\
(\%)\end{array}$ & $\begin{array}{l}\text { Uptake } \\
\text { (mg } \\
\left.\text { plant }^{-1}\right)\end{array}$ & Conc. (\%) & $\begin{array}{l}\text { Uptake } \\
\text { (mg } \\
\left.\text { plant }^{-1}\right)\end{array}$ & Conc. (\%) & $\begin{array}{l}\text { Uptake } \\
\text { (mg } \\
\left.\text { plant }^{-1}\right)\end{array}$ & Conc. (\%) & $\begin{array}{l}\text { Uptake } \\
\text { (mg } \\
\text { plant }^{-1} \text { ) }\end{array}$ \\
\hline $\mathrm{T}_{1}$ : Control (-RB and -NPK) & 2.18 & 152.6 & 0.28 & 19.6 & 2.56 & 179.2 & 0.10 & 7.0 \\
\hline $\mathrm{T}_{2}: 2.5$ t RB ha-1 & 2.33 & 233.0 & 0.28 & 28.0 & 2.80 & 280.0 & 0.13 & 13.0 \\
\hline $\mathrm{T}_{3}: 5.0$ t RB ha-1 & 2.36 & 212.4 & 0.31 & $27 \cdot 9$ & 3.41 & 306.9 & 0.15 & 13.5 \\
\hline $\mathrm{T}_{4}: 7.5 \mathrm{t} \mathrm{RB} \mathrm{ha}^{-1}$ & 2.37 & 237.0 & 0.34 & 34.0 & 3.43 & 343.0 & 0.17 & 17.0 \\
\hline $\mathrm{T}_{5}: \mathrm{N}_{40} \mathrm{P}_{30} \mathrm{~K}_{50} \mathrm{~kg} \mathrm{ha}^{-1}$ & 2.50 & 382.5 & 0.33 & 50.5 & 3.24 & $495 \cdot 7$ & 0.08 & 12.0 \\
\hline $\mathrm{T}_{6}: \mathrm{N}_{80} \mathrm{P}_{60} \mathrm{~K}_{100} \mathrm{~kg} \mathrm{ha}^{-1}$ & 2.62 & $453 \cdot 3$ & 0.34 & 58.8 & 3.49 & 603.8 & 0.12 & 20.8 \\
\hline $\mathrm{T}_{7}: \mathrm{N}_{120} \mathrm{P}_{90} \mathrm{~K}_{150} \mathrm{~kg} \mathrm{ha}^{-1}$ & 2.75 & 624.3 & 0.35 & 79.5 & 4.28 & 971.6 & 0.09 & 20.4 \\
\hline $\begin{array}{l}\text { T8: } 2.5 \mathrm{t} \mathrm{RB} \mathrm{ha}^{-1} \\
+\mathrm{N}_{40} \mathrm{P}_{30} \mathrm{~K}_{50} \mathrm{~kg} \mathrm{ha}^{-1}\end{array}$ & 2.79 & 521.7 & 0.39 & 72.9 & 4.16 & 777.9 & 0.12 & 22.4 \\
\hline $\begin{array}{l}\mathrm{T}_{9}: 2.5 \mathrm{t} \mathrm{RB} \mathrm{ha}^{-1} \\
+\mathrm{N}_{80} \mathrm{P}_{60} \mathrm{~K}_{100} \mathrm{~kg} \mathrm{ha}^{-1}\end{array}$ & 2.97 & 614.8 & 0.35 & 72.5 & 3.01 & 623.1 & 0.14 & 29.0 \\
\hline $\begin{array}{l}\mathrm{T}_{10}: 2.5 \mathrm{t} \mathrm{RB} \mathrm{ha}^{-1} \\
+\mathrm{N}_{120} \mathrm{P}_{90} \mathrm{~K}_{150} \mathrm{~kg} \mathrm{ha}^{-1}\end{array}$ & 3.17 & 634.0 & 0.36 & 72.0 & 4.13 & 826.0 & 0.16 & 32.0 \\
\hline $\begin{array}{l}\mathrm{T}_{11}: 5.0 \mathrm{t} \mathrm{RB} \mathrm{ha-1} \\
+\mathrm{N}_{40} \mathrm{P}_{30} \mathrm{~K}_{50} \mathrm{~kg} \mathrm{ha}^{-1}\end{array}$ & 3.10 & 536.3 & 0.34 & 58.8 & 3.59 & 621.1 & 0.18 & 31.1 \\
\hline $\begin{array}{l}\mathrm{T}_{12}: 5.0 \mathrm{t} \mathrm{RB} \mathrm{ha} \mathrm{H}^{-1} \\
+\mathrm{N}_{80} \mathrm{P}_{60} \mathrm{~K}_{100} \mathrm{~kg} \mathrm{ha}^{-1}\end{array}$ & 3.12 & 645.8 & 0.30 & 62.1 & 3.68 & 761.8 & 0.22 & $45 \cdot 5$ \\
\hline $\begin{array}{l}\mathrm{T}_{13}: 5.0 \mathrm{t} \mathrm{RB} \mathrm{ha} \mathrm{H}^{-1} \\
+\mathrm{N}_{120} \mathrm{P}_{90} \mathrm{~K}_{150} \mathrm{~kg} \mathrm{ha}^{-1}\end{array}$ & 3.98 & 903.5 & 0.43 & 97.6 & 4.07 & 923.9 & 0.23 & 52.2 \\
\hline $\begin{array}{l}\mathrm{T}_{14}: 7.5 \mathrm{t} \mathrm{RB} \mathrm{ha}^{-1} \\
+\mathrm{N}_{40} \mathrm{P}_{30} \mathrm{~K}_{50} \mathrm{~kg} \mathrm{ha}^{-1}\end{array}$ & 3.12 & 624.0 & 0.37 & 74.0 & 3.89 & 778.0 & 0.18 & 36.0 \\
\hline $\begin{array}{l}\mathrm{T}_{15}: 7.5 \mathrm{t} \mathrm{RB} \mathrm{ha}^{-1} \\
+\mathrm{N}_{80} \mathrm{P}_{60} \mathrm{~K}_{100} \mathrm{~kg} \mathrm{ha}^{-1}\end{array}$ & 3.96 & 650.4 & 0.38 & 91.2 & 3.74 & 897.6 & 0.20 & 48.0 \\
\hline $\begin{array}{l}\mathrm{T}_{16:} 7.5 \mathrm{t} \mathrm{RB} \mathrm{ha}^{-1} \\
+\mathrm{N}_{120} \mathrm{P}_{90} \mathrm{~K}_{150} \mathrm{~kg} \mathrm{ha}^{-1}\end{array}$ & 2.96 & 680.8 & 0.40 & 92.0 & 3.83 & 880.9 & 0.24 & 55.2 \\
\hline LSD at $5 \%$ & 0.54 & 21.59 & 0.06 & 7.34 & 0.57 & 35.15 & 0.05 & 4.76 \\
\hline
\end{tabular}

The highest values of both nitrogen and phosphorus were recorded in the same treatment, 5.0 t $\mathrm{RB} \mathrm{ha}^{-1}+\mathrm{N}_{120} \mathrm{P}_{90} \mathrm{~K}_{150} \mathrm{~kg} \mathrm{ha}^{-1}$ (Table 3). Similarly, concentration and uptake of potassium and sulfur in leaf ranged from 2.56 to $4.28 \%$ and 0.10 to $0.24 \%$; and 179.2 to 971.6 and 7.0 to 55.2 mg plant $^{-1}$ leaf, respectively (Table 3). The highest values were observed in treatments, $\mathrm{N}_{120} \mathrm{P}_{90} \mathrm{~K}_{150} \mathrm{~kg} \mathrm{ha}^{-1}$ for potassium and $7.5 \mathrm{t} \mathrm{RB} \mathrm{ha}^{-1}$ $+\mathrm{N}_{120} \mathrm{P}_{90} \mathrm{~K}_{150} \mathrm{~kg} \mathrm{ha}^{-1}$ for sulfur.

\section{(d) Concentration and uptake of NPKS in petiole}

Treatments of rice bran and NPK fertilizers, on concentration and uptake of NPKS in petiole were measured. Treatments showed significantly $(\mathrm{P}<0.05)$ positive effects with doses of rice bran and NPK fertilizers on nutrients concentrations of petiole of sunflower over the control (Table 4). But comparison between the treatments showed identical results. Between rice bran and fertilizers, rice bran played better role than inorganic fertilizers in case of only sulfur concentration. 
Table 4. Effects of rice bran compost and NPK fertilizers on the concentration and uptake of NPKS in petiole of sunflower.

\begin{tabular}{|c|c|c|c|c|c|c|c|c|}
\hline \multirow[t]{2}{*}{ Treatments } & \multicolumn{2}{|c|}{ Nitrogen } & \multicolumn{2}{|c|}{ Phosphorus } & \multicolumn{2}{|c|}{ Potassium } & \multicolumn{2}{|l|}{ Sulfur } \\
\hline & $\begin{array}{c}\text { Conc. } \\
\text { (\%) }\end{array}$ & $\begin{array}{l}\text { Uptake } \\
(\mathrm{mg} \\
\left.\text { plant }^{-1}\right)\end{array}$ & Conc. (\%) & $\begin{array}{l}\text { Uptake } \\
\text { (mg } \\
\left.\text { plant }^{-1}\right)\end{array}$ & Conc. (\%) & $\begin{array}{l}\text { Uptake } \\
(\mathrm{mg} \\
\left.\text { plant }^{-1}\right)\end{array}$ & Conc. (\%) & $\begin{array}{l}\text { Uptake } \\
\text { (mg } \\
\left.\text { plant }^{-1}\right)\end{array}$ \\
\hline $\mathrm{T}_{1}$ : Control (-RB and -NPK) & 0.53 & 6.9 & 0.14 & 1.8 & 0.56 & $7 \cdot 3$ & 0.04 & 0.5 \\
\hline $\mathrm{T}_{2}: 2.5$ t RB ha-1 & 0.57 & 9.1 & 0.20 & 3.2 & 0.83 & $13 \cdot 3$ & 0.12 & 1.9 \\
\hline $\mathrm{T}_{3}: 5.0$ t RB ha-1 & 0.61 & 18.3 & 0.25 & 7.5 & 1.31 & 39.3 & 0.16 & 4.8 \\
\hline $\mathrm{T}_{4}: 7 \cdot 5 \mathrm{t} \mathrm{RB} \mathrm{ha}^{-1}$ & 0.65 & 21.5 & 0.26 & 8.6 & 1.50 & 49.5 & 0.18 & $5 \cdot 9$ \\
\hline $\mathrm{T}_{5}: \mathrm{N}_{40} \mathrm{P}_{30} \mathrm{~K}_{50} \mathrm{~kg} \mathrm{ha}^{-1}$ & 0.97 & 34.0 & 0.29 & 10.2 & 1.62 & 56.7 & 0.07 & 2.5 \\
\hline $\mathrm{T}_{6}: \mathrm{N}_{80} \mathrm{P}_{60} \mathrm{~K}_{100} \mathrm{~kg} \mathrm{ha}^{-1}$ & 0.74 & 29.6 & 0.35 & 14.0 & 1.92 & 76.8 & 0.08 & 3.2 \\
\hline $\mathrm{T}_{7}: \mathrm{N}_{120} \mathrm{P}_{90} \mathrm{~K}_{150} \mathrm{~kg} \mathrm{ha}^{-1}$ & 0.79 & 43.5 & 0.42 & 23.1 & 1.99 & 109.5 & 0.09 & 5.0 \\
\hline $\begin{array}{l}\text { T8: } 2.5 \text { t RB ha-1 } \\
+\mathrm{N}_{40} \mathrm{P}_{30} \mathrm{~K}_{50} \mathrm{~kg} \mathrm{ha}^{-1}\end{array}$ & 0.63 & 27.1 & 0.44 & 18.9 & 1.66 & 71.4 & 0.11 & $4 \cdot 7$ \\
\hline $\begin{array}{l}\mathrm{T}_{9}: 2.5 \mathrm{t} \mathrm{RB} \mathrm{ha}^{-1} \\
+\mathrm{N}_{80} \mathrm{P}_{60} \mathrm{~K}_{100} \mathrm{~kg} \mathrm{ha}^{-1}\end{array}$ & 0.70 & 29.4 & 0.46 & $19 \cdot 3$ & 1.69 & 71.0 & 0.13 & $5 \cdot 5$ \\
\hline $\begin{array}{l}\mathrm{T}_{10}: 2.5 \mathrm{t} \mathrm{RB} \mathrm{ha}^{-1} \\
+\mathrm{N}_{120} \mathrm{P}_{90} \mathrm{~K}_{150} \mathrm{~kg} \mathrm{ha}^{-1}\end{array}$ & 0.85 & 40.8 & 0.41 & 19.7 & 2.13 & 102.2 & 0.16 & 7.7 \\
\hline $\begin{array}{l}\mathrm{T}_{11}: 5.0 \text { t RB ha } \\
+\mathrm{N}_{40} \mathrm{P}_{30} \mathrm{~K}_{50} \mathrm{~kg} \mathrm{ha}^{-1}\end{array}$ & 0.91 & 39.1 & 0.43 & 18.5 & 1.83 & 78.7 & 0.14 & 6.0 \\
\hline $\begin{array}{l}\mathrm{T}_{12}: 5.0 \text { t RB ha } \\
+\mathrm{N}_{80} \mathrm{P}_{60} \mathrm{~K}_{100} \mathrm{~kg} \mathrm{ha}^{-1}\end{array}$ & 0.95 & $45 \cdot 6$ & 0.49 & 23.5 & 1.89 & 90.7 & 0.17 & 8.2 \\
\hline $\begin{array}{l}\mathrm{T}_{13}: 5 \cdot 0 \text { t RB ha-1 } \\
+\mathrm{N}_{120} \mathrm{P}_{90} \mathrm{~K}_{150} \mathrm{~kg} \mathrm{ha}^{-1}\end{array}$ & 0.98 & 47.0 & 0.52 & 25.0 & 2.30 & 110.4 & 0.22 & 10.6 \\
\hline $\begin{array}{l}\mathrm{T}_{14}: 7.5 \mathrm{t} \mathrm{RB} \mathrm{ha} \\
+\mathrm{N}_{40} \mathrm{P}_{30} \mathrm{~K}_{50} \mathrm{~kg} \mathrm{ha}^{-1}\end{array}$ & 0.93 & 46.5 & 0.40 & 20.0 & 2.00 & 100.0 & 0.20 & 10.0 \\
\hline $\begin{array}{l}\mathrm{T}_{15}: 7.5 \text { t RB ha-1 } \\
+\mathrm{N}_{80} \mathrm{P}_{60} \mathrm{~K}_{100} \mathrm{~kg} \mathrm{ha}^{-1}\end{array}$ & 0.96 & $55 \cdot 7$ & 0.61 & 35.4 & 2.05 & 118.9 & 0.22 & 12.8 \\
\hline $\begin{array}{l}\mathrm{T}_{16}: 7.5 \mathrm{t} \mathrm{RB} \mathrm{ha}^{-1} \\
+\mathrm{N}_{120} \mathrm{P}_{90} \mathrm{~K}_{150} \mathrm{~kg} \mathrm{ha}^{-1}\end{array}$ & 1.04 & 59.3 & 0.65 & 37.1 & 3.00 & 171.0 & 0.19 & 10.8 \\
\hline LSD at $5 \%$ & 0.05 & 6.46 & 0.07 & 3.83 & 0.28 & 7.78 & 0.05 & 2.02 \\
\hline
\end{tabular}

The concentration and uptake of nitrogen and phosphorus in petiole ranged from 0.53 to 1.04 and 0.14 to $0.65 \%$, and 6.9 to 59.3 and 1.8 to 37.1 mg plant $^{-1}$ petiole, respectively (Table 4). The highest values for both concentration and uptake of nitrogen and phosphorus were observed in the same treatment, 7.5 $\mathrm{t} \mathrm{RB} \mathrm{ha-1}+\mathrm{N}_{120} \mathrm{P}_{90} \mathrm{~K}_{150} \mathrm{~kg} \mathrm{ha}^{-1}$. Like nitrogen and phosphorus, concentration and uptake of potassium and sulfur in petiole of sunflower varied from 0.56 to $3.00 \%$ and 0.04 to $0.22 \%$ and 7.3 to 171.0 and 0.5 to 12.8 mg plant $^{-1}$ petiole, respectively (Table 5 ). The highest values of concentration and uptake of potassium was found in same treatment, $7.5 \mathrm{t} \mathrm{RB} \mathrm{ha}+$ $\mathrm{N}_{120} \mathrm{P}_{90} \mathrm{~K}_{150} \mathrm{~kg} \mathrm{ha}^{-1}$. The highest values of concentration and uptake of sulfur was found in same treatment, 7.5 t RB ha-1 $+\mathrm{N}_{80} \mathrm{P}_{60} \mathrm{~K}_{100} \mathrm{~kg} \mathrm{ha}^{-1}$ here with in treatment, 5.0 t RB ha-1 $+\mathrm{N}_{120} \mathrm{P}_{90} \mathrm{~K}_{150}$ $\mathrm{kg} \mathrm{ha}^{-1}$ for sulfur concentration.

(e) Concentration and uptake of NPKS in inflorescence

The concentration and uptake of NPKS in inflorescence of sunflower were improved due to application of rice bran and NPK fertilizers in alone and various combinations. All the treatments increased the concentrations of NPKS in inflorescence of sunflower significantly $(\mathrm{P}<0.05)$ over the control (Table 5). Concentrations of NPKS increased with increasing doses of rice bran and NPK fertilizers. The differences between the treatments were identical in most of the cases. 
Table 5. Effects of rice bran compost and NPK fertilizers on the concentration and uptake of NPKS in inflorescence of sunflower.

\begin{tabular}{|c|c|c|c|c|c|c|c|c|}
\hline \multirow[t]{2}{*}{ Treatments } & \multicolumn{2}{|c|}{ Nitrogen } & \multicolumn{2}{|c|}{ Phosphorus } & \multicolumn{2}{|c|}{ Potassium } & \multicolumn{2}{|l|}{ Sulfur } \\
\hline & $\begin{array}{c}\text { Conc. } \\
(\%)\end{array}$ & $\begin{array}{l}\text { Uptake } \\
\text { (mg } \\
\text { plant }^{-1} \text { ) }\end{array}$ & Conc. (\%) & $\begin{array}{l}\text { Uptake } \\
\text { (mg } \\
\text { plant }^{-1} \text { ) }\end{array}$ & Conc. (\%) & $\begin{array}{l}\text { Uptake } \\
\text { (mg } \\
\text { plant }^{-1} \text { ) }\end{array}$ & Conc. (\%) & $\begin{array}{l}\text { Uptake } \\
\text { (mg } \\
\left.\text { plant }^{-1}\right)\end{array}$ \\
\hline $\mathrm{T}_{1}$ : Control (-RB and -NPK) & 0.36 & 14.4 & 0.09 & 3.6 & 0.75 & 30.0 & 0.05 & 2.0 \\
\hline $\mathrm{T}_{2}: 2.5$ t RB ha-1 & 0.39 & 20.7 & 0.16 & 8.5 & 1.02 & 54.1 & 0.11 & 5.8 \\
\hline $\mathrm{T}_{3}: 5.0$ t RB ha-1 & 0.58 & 50.5 & 0.18 & $15 \cdot 7$ & 1.16 & 100.9 & 0.15 & 13.1 \\
\hline $\mathrm{T}_{4}: 7 \cdot 5$ t RB ha-1 & 0.62 & 70.1 & 0.22 & 24.9 & 1.28 & 144.6 & 0.16 & 18.1 \\
\hline $\mathrm{T}_{5}: \mathrm{N}_{40} \mathrm{P}_{30} \mathrm{~K}_{50} \mathrm{~kg} \mathrm{ha}^{-1}$ & 1.42 & 132.1 & 0.27 & 25.1 & 1.40 & 130.2 & 0.06 & 5.6 \\
\hline $\mathrm{T}_{6}: \mathrm{N}_{80} \mathrm{P}_{60} \mathrm{~K}_{100} \mathrm{~kg} \mathrm{ha}^{-1}$ & 1.49 & 178.8 & 0.30 & 36.0 & 1.48 & 177.6 & 0.05 & 6.0 \\
\hline $\mathrm{T}_{7}: \mathrm{N}_{120} \mathrm{P}_{90} \mathrm{~K}_{15 \mathrm{o}} \mathrm{kg} \mathrm{ha}^{-1}$ & 1.55 & 227.9 & 0.37 & 54.4 & 1.56 & 229.3 & 0.07 & 10.3 \\
\hline $\begin{array}{l}\text { T8: } 2.5 \mathrm{t} \mathrm{RB} \mathrm{ha}^{-1} \\
+\mathrm{N}_{40} \mathrm{P}_{30} \mathrm{~K}_{50} \mathrm{~kg} \mathrm{ha}^{-1}\end{array}$ & 1.62 & 252.7 & 0.34 & $53 \cdot 3$ & 1.44 & 224.6 & 0.13 & 20.3 \\
\hline $\begin{array}{l}\mathrm{T}_{9}: 2.5 \mathrm{t} \mathrm{RB} \mathrm{ha}^{-1} \\
+\mathrm{N}_{80} \mathrm{P}_{60} \mathrm{~K}_{100} \mathrm{~kg} \mathrm{ha}^{-1}\end{array}$ & 1.70 & 287.3 & 0.39 & 65.9 & 1.52 & 256.9 & 0.14 & 23.7 \\
\hline $\begin{array}{l}\mathrm{T}_{10}: 2.5 \mathrm{t} \mathrm{RB} \mathrm{ha}^{-1} \\
+\mathrm{N}_{120} \mathrm{P}_{90} \mathrm{~K}_{150} \mathrm{~kg} \mathrm{ha}^{-1}\end{array}$ & 1.82 & 314.9 & 0.43 & 74.4 & 1.61 & 278.5 & 0.17 & 29.4 \\
\hline $\begin{array}{l}\mathrm{T}_{11}: 5.0 \mathrm{t} \mathrm{RB} \mathrm{ha-1} \\
+\mathrm{N}_{40} \mathrm{P}_{30} \mathrm{~K}_{50} \mathrm{~kg} \mathrm{ha}^{-1}\end{array}$ & 1.87 & 237.5 & 0.40 & 50.8 & 1.57 & 199.4 & 0.18 & 22.9 \\
\hline $\begin{array}{l}\mathrm{T}_{12}: 5.0 \mathrm{t} \mathrm{RB} \mathrm{ha}{ }^{-1} \\
+\mathrm{N}_{80} \mathrm{P}_{60} K_{100} \mathrm{~kg} \mathrm{ha}^{-1}\end{array}$ & 1.88 & 287.6 & 0.45 & 68.9 & 1.63 & 249.4 & 0.19 & 29.1 \\
\hline $\begin{array}{l}\mathrm{T}_{13}: 5.0 \text { t RB ha } \\
+\mathrm{N}_{120} \mathrm{P}_{90} \mathrm{~K}_{150} \mathrm{~kg} \mathrm{ha}^{-1}\end{array}$ & 2.03 & 365.4 & $0.5^{2}$ & 93.6 & 1.71 & 307.8 & 0.23 & 41.4 \\
\hline $\begin{array}{l}\mathrm{T}_{14}: 7.5 \mathrm{t} \mathrm{RB} \mathrm{ha-1} \\
+\mathrm{N}_{40} \mathrm{P}_{30} \mathrm{~K}_{50} \mathrm{~kg} \mathrm{ha}^{-1}\end{array}$ & 2.12 & 360.4 & 0.48 & 81.6 & 1.91 & 324.7 & 0.22 & 37.4 \\
\hline $\begin{array}{l}\mathrm{T}_{15}: 7.5 \mathrm{t} \mathrm{RB} \mathrm{ha}^{-1} \\
+\mathrm{N}_{80} \mathrm{P}_{60} \mathrm{~K}_{100} \mathrm{~kg} \mathrm{ha}^{-1}\end{array}$ & 2.14 & 256.8 & 0.54 & 64.8 & 2.09 & 250.8 & 0.25 & 30.0 \\
\hline $\begin{array}{l}\mathrm{T}_{16}: 7.5 \mathrm{t} \mathrm{RB} \mathrm{ha}^{-1} \\
+\mathrm{N}_{120} \mathrm{P}_{90} \mathrm{~K}_{150} \mathrm{~kg} \mathrm{ha}^{-1}\end{array}$ & 2.16 & 330.5 & 0.58 & 88.7 & 2.21 & 338.1 & 0.26 & 39.8 \\
\hline LSD at $5 \%$ & 0.05 & 27.45 & 0.05 & 7.28 & 0.05 & 29.95 & 0.43 & 3.60 \\
\hline
\end{tabular}

Nitrogen and phosphorus concentrations of inflorescence ranged from 0.36 to $2.16 \%$ and 0.09 to $0.58 \%$, respectively. The highest values were recorded in the same treatment $7.5 \mathrm{t} \mathrm{RB} \mathrm{ha}^{-1}$ $+\mathrm{N}_{120} \mathrm{P}_{90} \mathrm{~K}_{150} \mathrm{~kg} \mathrm{ha}^{-1}$. The values of uptake of nitrogen and phosphorus varied from 14.4 to 365.4 and 3.6 to $93.6 \mathrm{mg}$ inflorescence plant ${ }^{-1}$, respectively (Table 6). The highest uptake of nitrogen and phosphorus were recorded in same treatment, 5.0 t RB ha-1 $+\mathrm{N}_{120} \mathrm{P}_{90} \mathrm{~K}_{150} \mathrm{~kg} \mathrm{ha}^{-1}$. Potassium and sulfur concentrations were found to vary from 0.75 to $2.21 \%$ and 0.05 to $0.26 \%$, respectively (Table 5). Potassium and sulfur uptakes were found to range between 30.0 to 338.1 and 2.0 to $41.4 \mathrm{mg}$ plant $^{-1}$, respectively (Table 5). Highest values of both concentrations and uptakes of potassium and concentration of sulfur of inflorescence were being recorded in the same treatment, 7.5 $\mathrm{t} \mathrm{RB} \mathrm{ha}^{-1}+\mathrm{N}_{120} \mathrm{P}_{90} \mathrm{~K}_{150} \mathrm{~kg} \mathrm{ha}^{-1}$ and that of uptake of sulfur in 5.0 $\mathrm{t} \mathrm{RB} \mathrm{ha-1}+$ $\mathrm{N}_{120} \mathrm{P}_{90} \mathrm{~K}_{150} \mathrm{~kg} \mathrm{ha}^{-1}$ treatment.

\section{(f) Concentration and uptake of NPKS in seed}

The concentrations of NPKS in seed of sunflower were improved due to application of rice bran and NPK fertilizers in alone and various combinations. All the treatments increased the concentrations of NPKS in seed of sunflower significantly $(\mathrm{P}<0.05)$ over the control (Table 6). Concentrations of NPKS increased with increasing doses of rice bran and NPK fertilizers. The differences between the treatments were identical in most of the cases. Nitrogen and phosphorus concentrations and uptakes of seed showed a variation between 3.17 to 5.24 and 0.43 to $0.83 \%$, and 231.4 to 2396.6 and 31.4 to 345.0 mg plant ${ }^{-1}$ seed, respectively. The highest values of nitrogen and phosphorus concentrations were recorded in the treatments, 5.0 $\mathrm{t} \mathrm{RB} \mathrm{ha} \mathrm{R}^{-1}+$ $\mathrm{N}_{120} \mathrm{P}_{90} \mathrm{~K}_{150} \mathrm{~kg} \mathrm{ha}^{-1}$ and $7.5 \mathrm{t} \mathrm{RB} \mathrm{ha} \mathrm{h}^{-1}$, respectively. Similarly, the maximum values of uptake for both the nutrients were observed in the same treatment, $7.5 \mathrm{t} \mathrm{RB} \mathrm{ha}{ }^{-1}+\mathrm{N}_{80} \mathrm{P}_{60} \mathrm{~K}_{100} \mathrm{~kg} \mathrm{ha}^{-1}$. Concentrations and uptakes of potassium and sulfur in seeds of sunflower varied between 0.57 and 1.60, and 0.13 and $0.47 \% ; 41.6$ and 547.2 and 9.5 and 115.0 mg plant $^{-1}$ seed, respectively (Table 6). The highest values were observed in treatment, 2.5 t RB ha-1, and 5.0 t RB ha' 2.5t RB ha-1 $+\mathrm{N}_{40} \mathrm{P}_{30} \mathrm{~K}_{50} \mathrm{~kg} \mathrm{ha}^{-1}$ and 7.5 t RB ha ${ }^{-1}+$ $\mathrm{N}_{80} \mathrm{P}_{60} \mathrm{~K}_{100} \mathrm{~kg} \mathrm{ha}^{-1}$, respectively. 
Table 6. Effects of rice bran compost and NPK fertilizers on the concentration and uptake of NPKS and oil and protein content in seeds of sunflower.

\begin{tabular}{|c|c|c|c|c|c|c|c|c|c|c|}
\hline \multirow[t]{3}{*}{ Treatments } & \multicolumn{2}{|c|}{ Nitrogen } & \multicolumn{2}{|c|}{ Phosphorus } & \multicolumn{2}{|c|}{ Potassium } & \multicolumn{2}{|c|}{ Sulfur } & \multirow{2}{*}{$\begin{array}{l}\text { Oil } \\
\text { Con } \\
\text { tent }\end{array}$} & \multirow{2}{*}{$\begin{array}{l}\text { Protein } \\
\text { content }\end{array}$} \\
\hline & $\begin{array}{c}\text { Conc. } \\
\text { (\%) }\end{array}$ & $\begin{array}{l}\text { Uptake } \\
\text { (mg } \\
\left.\text { plant }^{-1}\right)\end{array}$ & $\begin{array}{l}\text { Conc. } \\
(\%)\end{array}$ & $\begin{array}{l}\text { Uptake } \\
(\mathrm{mg} \\
\left.\text { plant }^{-1}\right)\end{array}$ & $\begin{array}{l}\text { Conc. } \\
(\%)\end{array}$ & $\begin{array}{l}\text { Uptake } \\
(\mathrm{mg} \\
\left.\text { plant }^{-1}\right)\end{array}$ & $\begin{array}{l}\text { Conc. } \\
(\%)\end{array}$ & $\begin{array}{l}\text { Uptake } \\
(\mathrm{mg} \\
\left.\text { plant }^{-1}\right)\end{array}$ & & \\
\hline & & & & & & & & & $(\%)$ & (\%) \\
\hline $\mathrm{T}_{1}$ : Control (-RB and -NPK) & 3.17 & 231.4 & 0.43 & 31.4 & 0.57 & 41.6 & 0.13 & $9 \cdot 5$ & 40.5 & 18.9 \\
\hline $\mathrm{T}_{2}: 2.5$ t RB ha-1 & 3.21 & 343.5 & 0.53 & 56.7 & 1.60 & 171.2 & 0.22 & 23.5 & 39.0 & 19.8 \\
\hline $\mathrm{T}_{3}: 5.0$ t RB ha-1 & 3.23 & 474.8 & 0.68 & 100.0 & 1.39 & 204.3 & 0.47 & 69.1 & 44.8 & 20.0 \\
\hline $\mathrm{T}_{4}: 7.5 \mathrm{t} \mathrm{RB} \mathrm{ha}^{-1}$ & 3.27 & 631.1 & 0.83 & 160.2 & 1.14 & 220.0 & 0.23 & 44.4 & 43.7 & 21.2 \\
\hline $\mathrm{T}_{5}: \mathrm{N}_{40} \mathrm{P}_{30} \mathrm{~K}_{50} \mathrm{~kg} \mathrm{ha}^{-1}$ & 3.26 & 740.0 & 0.52 & 118.0 & 1.11 & 252.0 & 0.22 & 49.9 & $45 \cdot 4$ & 21.3 \\
\hline $\mathrm{T}_{6}: \mathrm{N}_{80} \mathrm{P}_{60} \mathrm{~K}_{100} \mathrm{~kg} \mathrm{ha}^{-1}$ & 3.27 & 981.0 & 0.57 & 171.0 & 1.11 & 333.0 & 0.21 & 63.0 & 45.2 & 21.7 \\
\hline $\mathrm{T}_{7}: \mathrm{N}_{120} \mathrm{P}_{90} \mathrm{~K}_{15 \mathrm{o}} \mathrm{kg} \mathrm{ha}^{-1}$ & 3.29 & 987.0 & 0.68 & 204.0 & 1.15 & 345.0 & 0.14 & 42.0 & 43.0 & 22.2 \\
\hline $\begin{array}{l}\mathrm{T}_{8}: 2.5 \mathrm{t} \mathrm{RB} \mathrm{ha}^{-1} \\
+\mathrm{N}_{40} \mathrm{P}_{30} \mathrm{~K}_{50} \mathrm{~kg} \mathrm{ha}^{-1}\end{array}$ & 3.39 & 1196.7 & 0.64 & 225.9 & 1.55 & 547.2 & 0.19 & 67.1 & 44.8 & 23.6 \\
\hline $\begin{array}{l}\mathrm{T}_{9}: 2.5 \mathrm{t} \mathrm{RB} \mathrm{ha}^{-1} \\
+\mathrm{N}_{80} \mathrm{P}_{60} \mathrm{~K}_{100} \mathrm{~kg} \mathrm{ha}^{-1}\end{array}$ & 3.86 & 1389.6 & 0.72 & 259.2 & 1.06 & 381.6 & 0.23 & 82.8 & $45 \cdot 3$ & 27.4 \\
\hline $\begin{array}{l}\mathrm{T}_{10}: 2.5 \mathrm{t} \mathrm{RB} \mathrm{ha}^{-1} \\
+\mathrm{N}_{120} \mathrm{P}_{90} \mathrm{~K}_{150} \mathrm{~kg} \mathrm{ha}^{-1}\end{array}$ & 4.04 & 1587.7 & 0.78 & 306.5 & 1.01 & 396.9 & 0.18 & 70.7 & 47.1 & 26.7 \\
\hline $\begin{array}{l}\mathrm{T}_{11}: 5.0 \text { t RB ha-1 } \\
+\mathrm{N}_{40} \mathrm{P}_{30} \mathrm{~K}_{50} \mathrm{~kg} \mathrm{ha}^{-1}\end{array}$ & $4 \cdot 34$ & 1532.0 & 0.65 & 229.5 & 1.15 & 406.0 & 0.19 & 67.1 & 48.7 & 27.9 \\
\hline $\begin{array}{l}\mathrm{T}_{12}: 5.0 \text { t RB ha } \\
+\mathrm{N}_{80} \mathrm{P}_{60} \mathrm{~K}_{100} \mathrm{~kg} \mathrm{ha}^{-1}\end{array}$ & 4.46 & 1306.8 & 0.75 & 219.8 & 0.96 & 281.3 & 0.19 & $55 \cdot 7$ & 45.2 & 33.9 \\
\hline $\begin{array}{l}\mathrm{T}_{13}: 5.0 \mathrm{t} \mathrm{RB} \mathrm{ha} \mathrm{Ra}^{-1} \\
+\mathrm{N}_{120} \mathrm{P}_{90} \mathrm{~K}_{150} \mathrm{~kg} \mathrm{ha}^{-1}\end{array}$ & 5.24 & 1991.2 & 0.82 & 311.6 & 1.15 & 437.0 & 0.29 & 110.2 & 51.1 & 31.0 \\
\hline $\begin{array}{l}\mathrm{T}_{14}: 7.5 \mathrm{t} \mathrm{RB} \mathrm{ha}^{-1} \\
+\mathrm{N}_{40} \mathrm{P}_{30} \mathrm{~K}_{50} \mathrm{~kg} \mathrm{ha}^{-1}\end{array}$ & 4.34 & 1562.4 & 0.69 & 248.4 & 1.39 & 500.4 & 0.22 & 79.2 & 43.8 & 30.5 \\
\hline $\begin{array}{l}\mathrm{T}_{15}: 7.5 \mathrm{t} \mathrm{RB} \mathrm{ha}^{-1} \\
+\mathrm{N}_{80} \mathrm{P}_{60} \mathrm{~K}_{100} \mathrm{~kg} \mathrm{ha}^{-1}\end{array}$ & 5.21 & 2396.6 & 0.75 & 345.0 & 1.15 & 529.0 & 0.25 & 115.0 & 49.8 & 28.9 \\
\hline $\begin{array}{l}\mathrm{T}_{16}: 7.5 \mathrm{t} \mathrm{RB} \mathrm{ha}^{-1} \\
+\mathrm{N}_{120} \mathrm{P}_{90} \mathrm{~K}_{150} \mathrm{~kg} \mathrm{ha}^{-1}\end{array}$ & 4.34 & 1245.6 & 0.64 & 183.7 & 1.25 & 358.8 & 0.23 & 66.0 & 46.3 & 25.8 \\
\hline LSD at $5 \%$ & 0.46 & 31.05 & 0.05 & 30.49 & 0.26 & 31.95 & 0.14 & 9.32 & 1.30 & 4.42 \\
\hline
\end{tabular}

These findings are in consistent with the observations of Badar and Qureshi (2014) who reported that composted rice husk improved mineral nitrogen and phosphorus contents of sunflower plants. Yankaraddi et al. (2009) also showed that application of FYM @ $10 \mathrm{t} \mathrm{ha}^{-1}+$ rice hull ash@2 t ha h $^{-1}$ 100\% RDF recorded the highest nutrient content $(2.51 \% \mathrm{~N}, 0.61 \% \mathrm{P}$ and 2.91\% K) and nutrient uptake (170.66 N, $41.14 \mathrm{P}$ and $209.76 \mathrm{~K} \mathrm{~kg} \mathrm{ha-1)}$ in rice plant. In this context, Akter et al. (2017), further, reported that the primary nutrition (NPK) of rice had better response in saline soil, which received rice hull, rice straw and saw dust. Marr and Cresser (1983) concluded that the typical concentrations of elements in dried healthy foliage are $\mathrm{N} \mathrm{0.8-3.0 \% ,}$ $\mathrm{K}$ 0.5-2.5\%, Ca 1.5-2.8\%, Mg 0.15-0.45\%, P 0.08$0.35 \%$, Fe 40-150 mg kg-1, Mn 30-100 mg kg-1, B 10-50 mg kg-1, Cu 5-12 mg kg-1, Zn 30-200 mg kg1 and Mo 0.1-1.5 mg kg-1. The result of the concentration of $\mathrm{N}, \mathrm{P}$ and $\mathrm{K}$ of the present experiment are in agreement with Marr and Cresser (1983).

\section{(g) Oil and protein content in seed}

Application of rice bran and NPK fertilizers at different combinations influenced on the oil content of sunflower seeds significantly $(\mathrm{P}<0.05)$ over the control except in treatment, $2.5 \mathrm{t} \mathrm{ha}^{-1}$ RB (Table 6). Results showed that all the treatments increased the oil content of seed following the increase of rice bran and NPK fertilizers except $2.5 \mathrm{t} \mathrm{ha}^{-1} \mathrm{RB}$. The variations among the treatments were found to be significant in most of the cases. The highest (51.1\%) content of oil was observed in 5.0 $\mathrm{t} \mathrm{RB}$ ha${ }^{1}+\mathrm{N}_{120} \mathrm{P}_{90} \mathrm{~K}_{150} \mathrm{~kg} \mathrm{ha}^{-1}$ treatment. The lowest (39.0\%) content of oil was observed in $2.5 \mathrm{t} \mathrm{RB}$ $\mathrm{ha}^{-1}$ treatment that the value was lower than control treatment.

These results are in agreement with the finding of Rasool et al. (2013) reported that application of organic manure @ 10 and $20 \mathrm{t} \mathrm{ha}^{-1}$ increased the oil yield of sunflower by 11 and 5.4\%, respectively, over no application of FYM. The author, further, revealed that with increased $\mathrm{N}$ dose, the oil content consistently decreased but the oil yield of sunflower improved by the application of FYM with $\mathrm{N}$ in two experiments. Similarly, Mahrous et al. (2014) reported that, there were no significant effect of the interaction between the varieties and various form of fertilizers (organic, bio and mineral) application in most of the studied traits except in seed oil content of sunflower. 
Protein content of sunflower seed also showed almost similar trend as in case of oil (Table 6). The treatments showed a gradual increase in protein content with the increase of doses of both the rice bran and NPK fertilizers. Six treatments namely 2.5 t RB ha-1, 5.0 t RB ha-1, 7.5 t RB ha-1, $\mathrm{N}_{40} \mathrm{P}_{30} \mathrm{~K}_{50} \mathrm{~kg} \mathrm{ha}^{-1}, \mathrm{~N}_{80} \mathrm{P}_{60} \mathrm{~K}_{100} \mathrm{~kg}$ ha-1 and $\mathrm{N}_{120} \mathrm{P}_{90} \mathrm{~K}_{150} \mathrm{~kg} \mathrm{ha}^{-1}$ showed not significant decrease in protein content when compared with the control. However, the rest of the treatments significantly $(\mathrm{P}<0.05)$ increased the protein content of sunflower seeds. The highest (33.9\%) and the lowest (18.9\%) contents of protein were recorded in 5.0 t $\mathrm{RB}$ ha $^{-1}+\mathrm{N}_{80} \mathrm{P}_{60} \mathrm{~K}_{100} \mathrm{~kg} \mathrm{ha}^{-1}$ and control treatments, respectively. Moreover, the variations among the treatments were found to be insignificant in most of the treatments as far as protein content was concerned. These findings agreed well with Badar and Qureshi (2014) who reported that decomposed rice husk improved total carbohydrate and protein contents of sunflower, which may be due to improvement in availability in nitrogen in soil. Similar information was also put forwarded by Mahrous et al. (2014).

\section{References}

Akter, S., Khan, M.H.R. and Hossain, M.S. 2017. Effects of rice hull, rice straw and saw dust application on the primary nutrients of rice plants growth under variable moisture conditions in a saline soil. Bangladesh J. Sci.

Res. 30(1\&2): 11-21.

https://doi.org/10.3329/bjsr.v3oi1-2.36116

AOAC. 1990. AOAC Official Methods of Analysis, $15^{\text {th }}$ edn. Association of Official Analytical Chemists. Arlington, Virginia, USA. pp. 8485 .

Badar, R. and Qureshi, S.A. 2014. Composted rice husk improves the growth and biochemical parameters of sunflower plants. J. Bot. 10(1): 1-6.

https://doi.org/10.1155/2014/427648

BARC. 2012. Fertilizer Recommendation Guide, BARC Soils Publication No. 49. Bangladesh Agricultural Research Council. People Press and Publications, Purana Palton, Dhaka. p. 112.

Bardsley, C.E. and Lancaster, J.D. 1965. Sulfur. In: Methods of Soil Analysis. Part 2. Black, C.A. (ed). ASA, Inc., Madison, Wisconsin, USA. pp. 1102-1114.
Bouyoucos, G.J. 1962. Hydrometer method improved for making particle size analysis of soils. Agron. J. 54: 461-465.

https://doi.org/10.2134/agronj1962.0002196200 $5400050028 \mathrm{x}$

Jackson, M.L. 1958. Soil Chemical Analysis. Prentice-Hall, Inc., Englewood cliffs, N.J. USA. p. 498.

Mahapatra, B.S. and Sharma, G.L. 1989. Integrated management of Sesbania, Azolla and urea nitrogen in low land rice- wheat cropping system. J. Agric. Sci. 113: 203-206. https://doi.org/10.1017/Soo21859600086779

Mahrous, N.M., Ragab, A.A., Abotaleb, H.H., Taha, M.H. and El-Metwally, M.S. 2014. Effect of inorganic, organic and biofertilizers on yield and yield components of sunflower under newly reclaimed soils. $J$. Plant Prod. Mansoura Univ. 5(3): 427-441. https://doi.org/10.21608/jpp.2014.53660

Marr, I.L. and Cresser, M.S. 1983. The lithosphere. In: Environmental Chemical Analysis. Blackie \& Son LTD. UK. pp. 155182.

Moyin-Jesu, E.I. 2015. Use of different organic fertilizers on soil fertility improvement, growth and head yield parameters of cabbage (Brassica oleraceae L.). Int. J. Recl. Org. Waste Agric. 4: 291-298.

https://doi.org/10.1007/s40093-015-0108-0

Pratt, P.F. 1965. Potassium. In: Method of Soil Analysis. Part 2, Black, C.A. (ed). SAA Inc., Madison, Wisconsin, pp. 1022-1030.

https://doi.org/10.2134/agronmonogr9.2.c20

Rasool, F.U., Hassan, B. and Jahangir, I.A. 2013. Growth and yield of sunflower (Helianthus annuus L.) as influenced by nitrogen, sulphur and farmyard manure under temperate conditions. SAARC J. Agric. 11(1): 81-89.

https://doi.org/10.3329/sja.v11i1.18386

Walkley, A. and Black, C.A. 1934. An examination of the degtjareff method for determining soil organic matter and a proposed modification for the chromic acid titration method. Soil Sci. 37: 29-38.

https://doi.org/10.1097/00010694-19340100000003

Yankaraddi, H., Dinesh, K.M. and Madaiah, D. 2009. Effect of coffee pulp compost and rice hull ash on growth, yield and nutrient uptake in rice. Karnataka J. Agric. Sci., 22(4): 751-754. 